\title{
IgG Endopeptidase SeMac does not Inhibit Opsonophagocytosis of Streptococcus equi Subspecies equi by Horse Polymorphonuclear Leukocytes
}

\author{
Mengyao Liu and Benfang Lei*
}

Department of Veterinary Molecular Biology, Montana State University, Bozeman, Montana 59717, USA

\begin{abstract}
The secreted Mac protein made by group A Streptococcus (GAS) inhibits opsonophagocytosis of GAS by human polymorphonuclear leukocytes (PMNs). This protein also has the endopeptidase activity against human immunoglobulin G (IgG), and the Cys94, His262 and Asp284 are critical for the enzymatic activity. The horse pathogen Streptococcus equi subspecies equi produces a homologue of Mac (SeMac). SeMac was characterized to determine whether SeMac has IgG endopeptidase activity and inhibits opsonophagocytosis of S. equi by horse PMNs. The gene was cloned and recombinant SeMac was overexpressed in Escherichia coli and purified to homogeneity. Mice with experimental $S$. equi infection and horses with strangles caused by $S$. equi seroconverted to SeMac, indicating that SeMac is produced in vivo during infection. SeMac has endopeptidase activity against human IgG. However, the protein just cleaves a small fraction, which may be IgG1 only, of horse IgG. Replacement of Cys102 with Ser or His272 with Ala abolishes the enzymatic activity of SeMac, and the Asp294Ala mutation greatly decreases the enzymatic activity. SeMac does not inhibit opsonophagocytosis of $S$. equi by horse PMNs but opsonophagocytosis of GAS by human PMNs. Thus, SeMac is a cysteine endopeptidase with a limited activity against horse IgG and must have other function.
\end{abstract}

Keywords: IgG, Endopeptidase SeMac, opsonophagocytosis, polymorphonuclear leukocytes.

\section{INTRODUCTION}

Gram-positive bacterium Streptococcus equi subspeices equi (S. equi) causes equine strangles, a highly contagious purulent lymphadenitis and one of the most common infectious diseases in horses [1,2]. The infection initially causes nasal discharge and fever and, then, leads to abscess formation in local lymph nodes, causing enormous pain and respiratory difficulty. There is massive infiltration of polymorphonuclear leukocytes (PMNs) to the infection site. However, $S$. equi effectively evades the horse innate immunity by being resistant to phagocytosis by PMNs. Horses recovered from strangles acquire immunity against $S$. equi reinfection [3]. The immunity is primarily mediated by protective antibodies [4], which opsonize and thus enhance phagocytosis of S. equi by horse PMNs.

To survive in hosts, bacterial pathogens have evolved multiple mechanisms to evade host defense. For examples, both S. equi and group A Streptococcus (GAS) produce the hyaluronic acid capsule and surface protein $M$ protein to contribute to resistance to phagocytosis by PMNs. We found that GAS produces a secreted Mac protein (also known as IdeE), which inhibits opsonophagocytosis of GAS by human PMNs [5]. This protein can cleave the heavy chain of human immunoglobulin $\mathrm{G}$ ( $\mathrm{IgG}$ ) using Cys94, His262 and Asp284 as its catalytic triad [6-8]. There are two kinds of Mac

*Address correspondence to this author at the Department of Veterinary Molecular Biology, Montana State University, P.O. Box 173610, Bozeman, Montana 59717, USA; Tel: 406-994-6389; Fax: 406-994-4303;

E-mail: blei@montana.edu produced by GAS isolates [7], which use different mechanisms to block the interaction between $\mathrm{IgG}$ and $\mathrm{Fc}$ receptor on the surface of PMNs. The type-1 Mac, such as M1 Mac produced by serotype M1 GAS strains, has high enzymatic activity and low affinity to $\mathrm{Fc}$ receptor on the surface of PMNs, while the type- 2 Mac can bind to the Fc receptor and has lower enzymatic activity [9]. S. equi has a homologue of GAS M1 Mac (designated SeMac). In this study, SeMac was prepared and characterized. The results indicate that SeMac is a cysteine endopeptidase but does not inhibit opsonophagocytosis of $S$. equi by horse PMNs, suggesting that SeMac has function other than evading horse acquired immunity against $S$. equi infection.

\section{MATERIALS AND METHODS}

\section{Materials}

Purified horse IgG1 and a mixture of horse IgG1 and IgG4 were kindly provided by Dr. Bettina Wagner at Cornell University. S. equi-specific mouse sera were obtained from adult female outbred CD-1 Swiss mice (Charles River Laboratories, Wilmington, Mass.) 21 days after they were inoculated subcutaneously with $1 \times 10^{7}$ cfu S. equi strain SEM1. Convalescent sera from 3 horses suffering from strangles were obtained 30 days after diagnosis. GAS M1 Mac was prepared as previously described [5].

\section{Bacterial Strains and Growth}

Six of $10 S$. equi strains used were kindly provided by Dr. James Musser at Methodist Hospital, Houston, Texas, 
and these strains were isolated more than 20 years ago from horses with strangles in the Eastern U.S. (4 strains), Brazil (1 strain), and Finland (1 strain). The other 4 strains were isolated in 2003 from horses with strangles in Livingston (designated strain SEM1) [10], Pony, Great Fall, and Norris in Montana. GAS strain MGAS5005 (serotype M1) has been described [11]. Escherichia coli Novablue and BL21(DE3) (Novagen, Madison, Wis.) were used for gene cloning and protein expression, respectively. S. equi and GAS strains were routinely grown in Todd-Hewitt broth (Difco Laboratories, Detroit, Mich.) supplemented with $0.2 \%$ yeast extract (THY) in $5 \% \mathrm{CO}_{2}$ at $37^{\circ} \mathrm{C}$. THY agar and tryptose agar with $5 \%$ sheep blood (Becton Dickinson, Cockeysville, Md.) were used as the solid media.

\section{Gene Cloning and Mutagenesis}

The gene fragment encoding mature SeMac was cloned from SEM1 with primers 5'-ACCATGGACGA TTACCAAAGGAATGCTAC-3' and 5'-CGAATTCT TAGCTCAGTTTCTGCCATATG-3'. The protein made from this cloned fragment lacks the presumed secretion signal sequence (amino acids 1-34). The PCR product was digested with $N c o$ I and EcoRI and ligated into pET-21d (Novagen) to yield recombinant plasmid pSEMAC. The cloned gene was sequenced and had identical DNA sequence with the corresponding open reading frame of $S$. equi genome database [12]. Amino acid replacement of Cys102 and His272 or Asp294 of SeMac with serine and alanine, respectively, was achieved by site-directed mutagenesis using the QuickChange Mutagenesis kit (Stratagene, La Jolla, Calif.). The entire mutated gene was sequenced to confirm the mutations and rule out spurious mutations.

\section{Expression and Purification of Recombinant SeMac}

Recombinant SeMac was purified from E. coli BL21 (DE3) containing plasmid pSEMAC. Bacteria were grown to optical density at $600 \mathrm{~nm}$ of 0.5 in 6 liters of Luria-Bertani broth supplemented with $100 \mathrm{mg}$ of ampicillin per liter at $37^{\circ} \mathrm{C}$, and SeMac expression was then induced with $0.5 \mathrm{mM}$ IPTG $6 \mathrm{~h}$. Solutions used in purification were buffered with $10 \mathrm{mM}$ Tris- $\mathrm{HCl}(\mathrm{pH} \mathrm{8.0)}$. Cell paste was sonicated for 15 $\min$ at $4^{\circ} \mathrm{C}$ in $60 \mathrm{ml}$ of the buffer, and centrifuged at 15,000 $\mathrm{g}$ for $10 \mathrm{~min}$. The supernatant obtained was loaded onto a DEAE-Sepharose column $(2.5$ by $10 \mathrm{~cm})$. The column was washed with $50 \mathrm{ml}$ of Tris-HCl, and SeMac was eluted with $200 \mathrm{ml}$ of $50 \mathrm{mM} \mathrm{NaCl}$. SeMac was identified by sodium dodecyl sulfate-polyacrylamide gel electrophoresis (SDSPAGE), and peak fractions were pooled. Ammonium sulfate was added to the pool to a concentration of $1.2 \mathrm{M}$, and the resulting solution was applied to a phenyl-Sepharose column $(1.5 \times 10 \mathrm{~cm})$. The column was washed with $50 \mathrm{ml}$ of $1.2 \mathrm{M}$ $\left(\mathrm{NH}_{4}\right)_{2} \mathrm{SO}_{4}$ and eluted with a $100-\mathrm{ml}$ linear gradient of 1.2 to $0.7 \mathrm{M}\left(\mathrm{NH}_{4}\right)_{2} \mathrm{SO}_{4}$. Fractions containing the protein were pooled. The pooled protein was precipitated with $\left(\mathrm{NH}_{4}\right)_{2} \mathrm{SO}_{4}$ at $70 \%$ of saturation, centrifuged, dialyzed against 3 liters of $10 \mathrm{mM}$ Tris- $\mathrm{HCl}$ buffer ( $\mathrm{pH} 8.0$ ) overnight, and loaded onto a DEAE-Sepharose column $(1.5$ by $10 \mathrm{~cm})$. The protein was eluted with a $100-\mathrm{ml}$ linear gradient of 2 to $8 \mathrm{mM} \mathrm{NaCl}$ and pooled. The pooled protein was concentrated by $\left(\mathrm{NH}_{4}\right)_{2} \mathrm{SO}_{4}$ precipitation and dialyzed against Tris- $\mathrm{HCl}$ as described above.

\section{DNA Sequencing}

Genomic DNA was isolated with a FastDNA SPIN Kit (Qbiogene, Calsdad, Calif.) according to manufacturer's protocol. A DNA fragment containing S. equi mac was amplified by PCR using the genomic DNA samples and primers 5'-GCATCTCTACTATCTCATCAC-3' and 5'-ACAGGCACATTAATGTTTAAC-3'. Sequences of the PCR products were obtained from both DNA strands with an Applied Biosystem 310 automated sequencer (Applied BioSystems, Inc., Foster City, Calif.).

\section{Anti-SeMac antisera}

Four female outbred CD-1 Swiss mice (4-week-old) (Charles River Laboratories) were immunized subcutaneously with $50 \mu \mathrm{g}$ of recombinant SeMac suspended in $200 \mu \mathrm{l}$ of saline emulsified in $44 \mu \mathrm{l}$ of monophosphoryl lipid Asynthetic trehalose dicorynomycolate adjuvant (Corixa, Hamilton, Mont.). The animals were anesthetized by isoflurane inhalation prior to immunization and blood collection. Blood was collected prior to active immunization to prepare control sera. Mice were boosted at weeks 2 and 4 with $50 \mu \mathrm{g}$ of protein mixed with the adjuvant. Anti-SeMac antiserum was prepared from blood obtained 1 week after the second booster.

\section{Phagocytosis Assay}

Phagocytosis assay was performed as described previously $[13,14]$. Briefly, S. equi SEM1 and GAS bacteria from exponential growth phase in THY were washed with phosphate-buffered saline (PBS) and labeled with $0.75 \mu \mathrm{g} / \mathrm{ml}$ FITC in PBS at $37^{\circ} \mathrm{C}$ for $20 \mathrm{~min}$. The labeled bacteria were washed and resuspended at $1 \times 10^{9} \mathrm{cfu} / \mathrm{ml}$ in PBS. Ten $\mu 1$ of the labeled bacteria were mixed with $100 \mu$ l of non-immune heparinized horse or human blood with or without $100 \mu \mathrm{g} / \mathrm{ml}$ SeMac and incubated with gentle shaking at $37^{\circ} \mathrm{C}$ for 5,10 , or $20 \mathrm{~min}$. The samples were immediately processed using an Immunolyse Kit (Beckman Coulter) according to the manufacturer's protocol and analyzed by flow cytometry. The percentage of PMNs with fluorescent bacteria was used as a measure of phagocytosis efficiency.

\section{Other Assays}

To assess IgG endopeptidase activity of SeMac, human, mouse or horse IgG $(20 \mu \mathrm{g})$ was incubated with E. coli lysate containing SeMac proteins or $1 \mu \mathrm{g}$ purified SeMac or GAS $\mathrm{M} 1 \mathrm{Mac}$ in $\mathrm{PBS}$ at $37^{\circ}$ for $90 \mathrm{~min}$, and the reaction mixture was analyzed by SDS-PAGE. Western immunoblot analysis, which was performed as described previously [15], was used to assess in vitro SeMac production and the presence of SeMac-specific antibody in the horse and mouse convalescent sera. Culture supernatant proteins of $S$. equi, which were used to assess the in vitro SeMac production, were prepared by the method of Lei et al. [16].

\section{RESULTS}

\section{S. equi Mac Gene}

S. equi genome encodes a Mac homologue SeMac. The protein shares $62.4 \%$ identity in amino acid sequence with Mac made by serotype M1 GAS and a putative secretion 
signal sequence (amino acids 1-34), and the catalytic residues of GAS Mac consisting of Cys94, His262 and Asp284 are conserved in SeMac (Cys102, His272 and Asp 294) (Fig. 1). To test whether other $S$. equi strains have the mac gene, $10 \mathrm{~S}$. equi isolates representing various geographic locations were tested with PCR using mac-specific primers. All the strains tested had the mac gene (Fig. 2). DNA sequencing found that the mac gene is $100 \%$ conserved in DNA sequence in these strains.

\section{Recombinant SeMac and In Vitro and In Vivo Expression of SeMac}

To characterize SeMac, the fragment of $S$. equi mac gene encoding mature SeMac was cloned, and recombinant SeMac was purified to $>95 \%$ purity as assessed by SDSPAGE (Fig. 3A). To assess the in vitro production of SeMac, culture supernatant of the $10 \mathrm{~S}$. equi strains was prepared by the method of Lei et al. [16], resolved by SDS-PAGE, and probed by Western immunoblot with anti-SeMac mouse antisera. No SeMac was detected in all the samples (data not shown), suggesting that SeMac is not produced in vitro. To test whether SeMac is produced in vivo during $S$. equi infection, the presence of SeMac-specific antibody was assessed by Western immunoblot analysis with convalescent sera from 3 horses suffered from strangles and mice with experimental $S$. equi infection. All the convalescent sera tested had SeMac-specific antibody (Fig. 3B), indicating that SeMac is produced in vivo during infection.

\section{Endopeptidase Activity of SeMac against Horse IgG1 and Human IgG}

GAS Mac can cleave the heavy chain of human IgG at the lower hinge region between Fab and Fc fragments. A catalytic triad of Cys94, His262, and Asp284 residues is critical for the enzymatic activity of GAS Mac [7, 8]. SeMac possesses putative catalytic residues of Cys102, His272, and Asp294 (Fig. 1). To determine whether SeMac also is a cysteine endopeptidase targeting IgG, Cys102 and His272 or Asp294 of SeMac were replaced with Ser and Ala, respectively, by site-directed mutagenesis, and wild-type and mutant (SeMac ${ }^{\text {Cys102Ser }}$, SeMac ${ }^{\text {His272Ala }}$ and SeMac ${ }^{\text {Asp294Ala }}$ ) SeMac proteins expressed in E. coli were tested for IgG endopeptidase activity using human IgG. Wild-type SeMac cleaved the heavy chain of human IgG, while SeMac Cys102Ser and SeMac ${ }^{\text {His272Ala }}$ completely lost the $\operatorname{IgG}$ endopeptidase activity, and SeMac ${ }^{\text {Asp294Ala }}$ had dramatically lower enzymatic activity than the wild-type protein (Fig. 4A). The results indicate that SeMac is a cysteine endopeptidase, which uses Cys102, His272 and Asp294 as its catalytic triad to cleave human IgG.

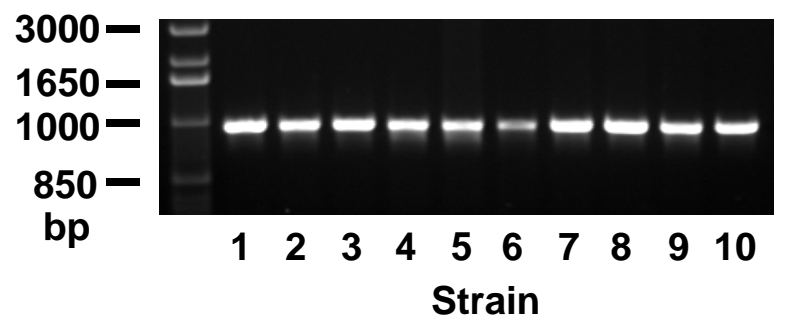

Fig. (2). Detection of $S$. equi mac gene in clinical isolates by PCR. The picture shows the mac PCR product amplified from $10 \mathrm{~S}$. equi isolates from horses suffering from strangles.
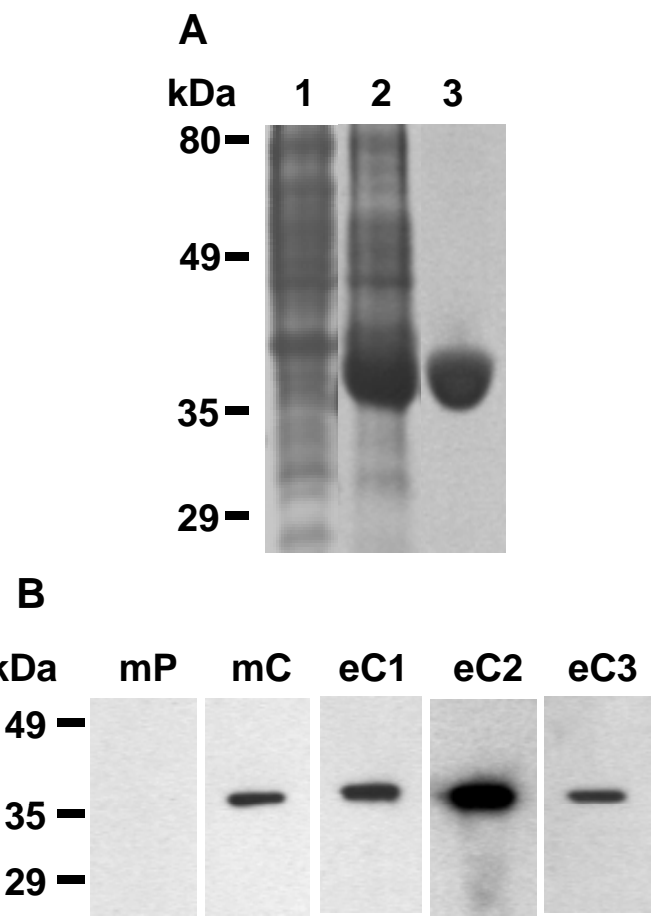

Fig. (3). Recombinant SeMac and evidence for in vivo production of SeMac. (A) SDS-PAGE analysis of recombinant SeMac. Lanes: 1, E. coli lysate without SeMac (control); 2, E. coli lysate containing overexpressed SeMac; 3, purified SeMac. (B) Western immunoblot analysis of SeMac with SeMac antibody present in the convalescent-phase sera from 3 horses with $S$. equi infection (eC1, $\mathrm{eC} 2, \mathrm{eC} 3)$ and mice before $(\mathrm{mP})$ and after $(\mathrm{mC})$ experimental $S$. equi infection.

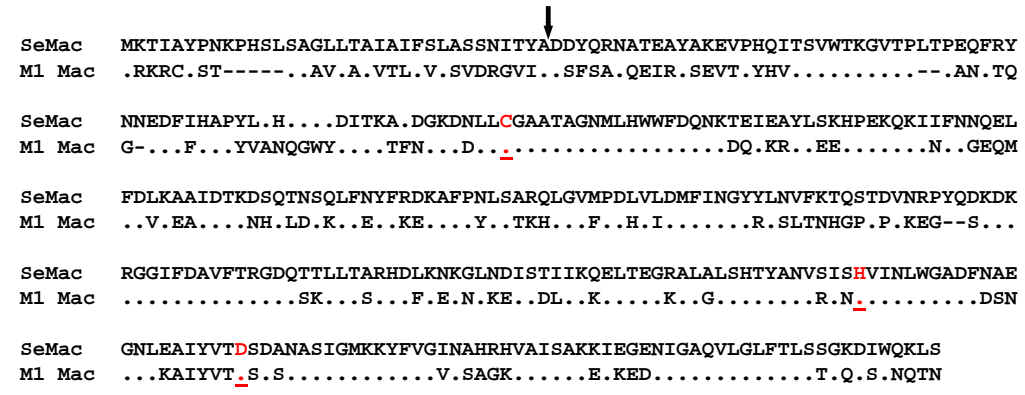

Fig. (1). Amino acid sequence alignment of SeMac and GAS M1 Mac. The conserved Cys (C), His (H), and Asp (D) constituting the catalytic triad are highlighted in red and underlined. Amino acid residues of GAS Mac identical to those in SeMac (.) and gaps introduced to maximize alignment (-) are indicated. The arrow indicates the presumed cleavage site of the secretion signal sequence. 
Purified recombinant SeMac and GAS Mac were then tested for activity to cleave horse IgG. While both proteins cleaved human IgG efficiently, only a small fraction of horse IgG was cleaved by either protein (Fig. 4B), suggesting that SeMac or GAS Mac cannot digest all subgroups of horse IgG. To test this possibility, available horse IgG1 and a mixture of IgG1 and IgG4 were treated with SeMac or GAS Mac. SeMac or GAS Mac cleaved a small portion of the IgG1/IgG4 mixture, but not IgG4. Therefore, the Mac proteins can cleave IgG1 but not IgG4. Due to the unavailability of other purified subgroup $\mathrm{IgG}$, it is not known whether SeMac cleaves the other IgG subgroups.

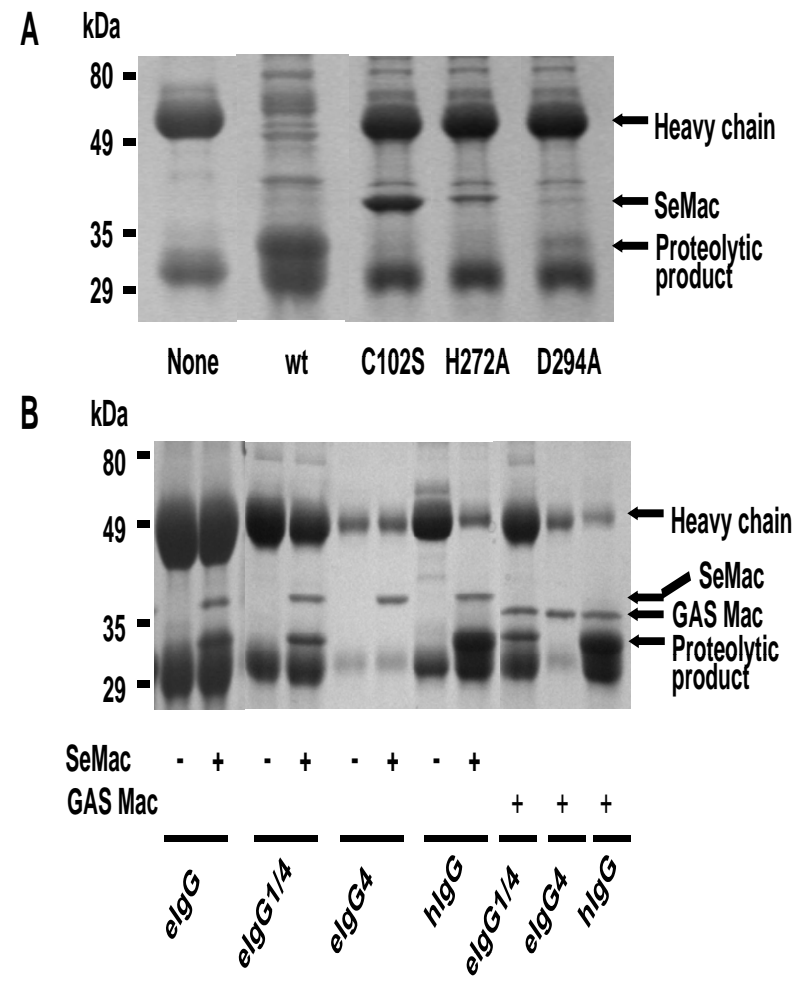

Fig. (4). IgG endopeptidase activity of SeMac. (A) IgGendopeptidase activity and critical amino acid residues for activity. Human IgG was incubated without (none) or with E. coli lysate containing wild-type SeMac (wt), SeMac ${ }^{\text {Cys102Ser }}$ (C102S), Se$\mathrm{Mac}^{\text {His272Ala }}$, or SeMac ${ }^{\text {Asp294Ala }}$ at room temperature for $60 \mathrm{~min}$ and analyzed by SDS-PAGE. (B) Limited cleavage of horse IgG by SeMac and GAS Mac. Horse total IgG (eIgG), IgG4 (eIgG4), IgG1/IgG4 mixture (eIgG1/4), or human IgG was incubated with purified SeMac or GAS M1 Mac and analyzed by SDS-PAGE.

\section{SeMac does not Inhibit Opsonophagocytosis of $S$. equi by Horse PMN}

A whole blood based phagocytosis assay [13, 14] was used to test whether SeMac inhibits opsonophagocytosis of $S$. equi by horse PMNs. The bacteria were labeled with FITC, treated with $S$. equi-specific horse convalescent serum in the absence or presence of SeMac, and incubated with horse blood. Percentage of PMNs with phagocytosed $S$. equi was determined by flow cytometry. As expected, percentage of PMNs with phagocytosed $S$. equi with the serum treatment was significantly higher than that for $S$. equi without serum treatment. But the inclusion of SeMac in the assay did not affect opsonophagocytosis of $S$. equi (Fig. 5A), suggest- ing that SeMac does not inhibit opsonophagocytosis of $S$. equi by horse PMNs.

The inability of SeMac to inhibit $S$. equi opsonophagocytosis by horse PMNs may be due to its inability to cleave the majority of horse IgG. If this is true, SeMac should inhibit opsonophagocytosis of GAS by human PMNs, since SeMac efficiently cleaves human IgG. To test this idea, the phagocytosis assay was repeated using GAS and human blood. SeMac indeed inhibits the opsonophagocytosis of GAS by human PMNs (Fig. 5B). These results suggest that IgG endopeptidase activity of SeMac is critical for inhibition of phagocytosis.

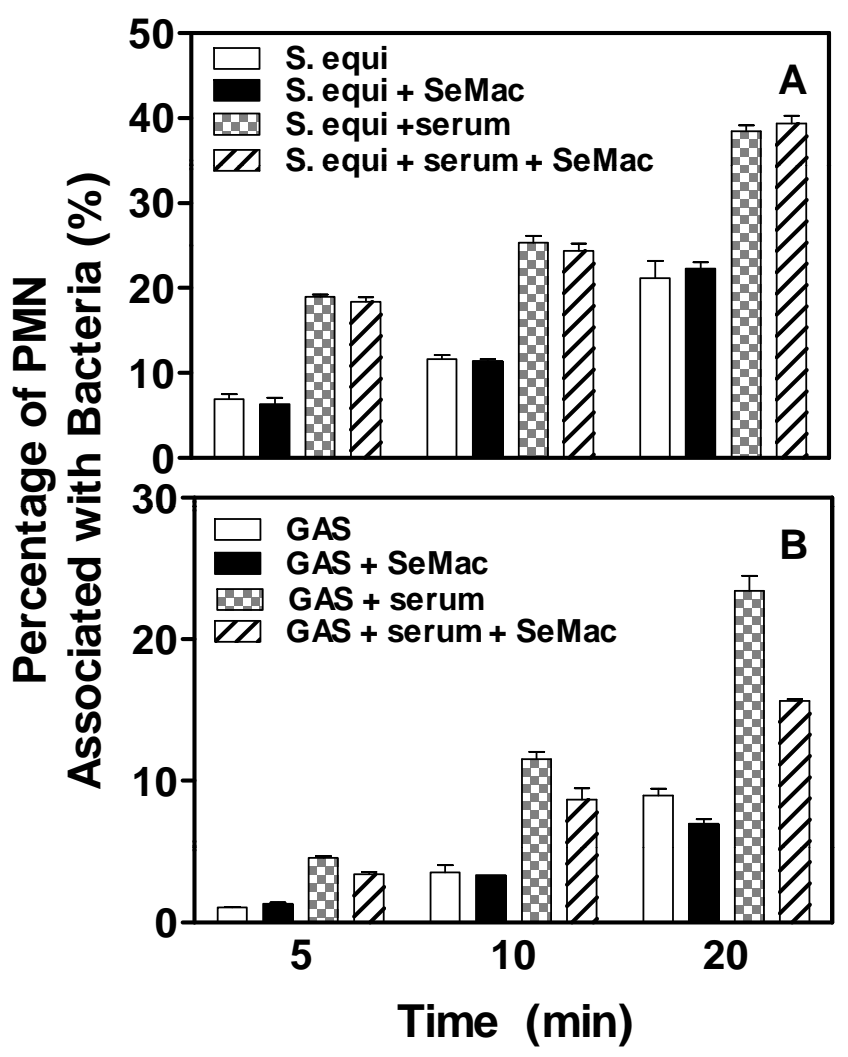

Fig. (5). Effects of SeMac on opsonophagocytosis of $S$. equi and GAS by horse (A) and human (B) PMNs, respectively. FITClabeled $S$. equi and GAS bacteria $\left(10^{7}\right.$ c.f.u.) were treated without or with horse and human convalescent sera and incubated in the presence or absence of $100 \mu \mathrm{g} / \mathrm{ml}$ SeMac with $100 \mu \mathrm{l}$ nonimmune horse and human blood, respectively, at $37^{\circ} \mathrm{C}$ for 5,10 or $20 \mathrm{~min}$. Red blood cells were lysed using an Immunolyse kit, and percentages of PMNs with phagocytosed bacteria determined by flow cytometry are presented.

\section{DISCUSSION}

In this study we describe that SeMac is a cysteine endopeptidase with limited activity against horse IgG and is unable to inhibit opsonophagocytosis of $S$. equi by horse PMNs in a whole blood assay. SeMac efficiently cleaves the heavy chain of human IgG. The residues of Cys102 and His272 are essential and Asp294 important for the enzymatic activity of SeMac. Thus, like GAS M1 Mac [7,8], SeMac is a cysteine endopeptidase. Although both SeMac and GAS Mac efficiently cleave human IgG, they cannot cleave the 
Table 1. The Amino Acid Sequences of the Lower Hinge Region of Human and Horse IgG Subgroups and Their Cleavability by GAS M1 Mac and SeMac

\begin{tabular}{|c|c|c|c|}
\hline \multirow[t]{2}{*}{ IgG Subgroup } & \multirow{2}{*}{$\begin{array}{c}\text { AA Sequence } \\
\text { in the Lower Hinge Region }{ }^{a}\end{array}$} & \multicolumn{2}{|c|}{ Cleaved by ${ }^{b}$} \\
\hline & & M1 Mac & SeMac \\
\hline Human IgG2 & VE......PVA-_... & + & + \\
\hline Human IgG3 & PP.R....... & + & + \\
\hline Horse IgG1 & sk. . KC......... & + & + \\
\hline Horse IgG2 & PQ. . YTHSKF . . . . . & ND & ND \\
\hline Horse IgG3 & 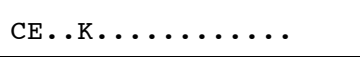 & ND & ND \\
\hline Horse $\operatorname{IgG7}$ & CGGC.TC..C.SV.... & ND & ND \\
\hline
\end{tabular}

${ }^{a}$ Cleavage was prior to the Gly residue underlined and determined by N-terminal amino acid sequencing of the proteolytic products in the reaction catalyzed by GAS M1, Mac and dots indicate identical residues with human IgG1.

${ }^{b}+$, cleaved; -, not cleaved; ND, not determined.

majority of total horse IgG. The peptide fragment, PELLGG, in the lower hinge region is proposed to bind to the active site of Mac [9], and the cleavage occurs between the two Gly residues (Table 1). This lower hinge region is not well conserved in the seven subgroups of horse IgG [17] (Table 1). Cleavable horse IgG1 has PELLGG, while the non-cleavable horse IgG4 has PECLSVG in the same region, suggesting that the amino acid sequences of the lower hinge region are important for cleavability by SeMac and GAS Mac. If this is true, horse IgG3 may be also cleavable by SeMac, while the other five horse IgG subgroups may not be cleaved. These horse IgG antibodies that cannot be cleaved by SeMac thus still can mediate the opsonophagocytosis of $S$. equi.

SeMac and GAS M1 Mac show similar enzymatic specificity (Fig. 4 and Table 1), confirming the previous finding [18]. SeMac can cleave human IgG and inhibit the opsonophagocytosis of GAS by human PMNs, but it has limited enzymatic activity against horse IgG and is unable to inhibit opsonophagocytosis of $S$. equi by horse PMNs. Thus, there is a correlation between the enzymatic activity of SeMac and its ability to inhibit opsonophagocytosis, suggesting that SeMac functions like GAS M1 Mac in the inhibition of opsonophagocytosis of GAS by human PMNs.

Timoney et al. recently reported that IdeE/SeMac reduces the bactericidal activity of isolated equine PMNs for $S$. equi [19]. Our results suggest that the inhibition of the bactericidal activity of PMNs may not be mediated by opsonophagocytosis or may be insignificant in whole blood.

SeMac is not produced in vitro, whereas GAS Mac is [16]. The mac gene is controlled by the two-component regulatory system CovRS [5], which also controls the expression of many virulence factors including the hyaluronic capsule [20]. The hyaluronic capsule of $S$. equi is highly produced in vitro. These observations suggest that the semac and mac genes are regulated by different mechanisms. This suggestion is supported by only $29 \%$ DNA sequence identity between $\mathrm{S}$. equi and M1 GAS in the upstream region of the semac and mac genes.

S. equi is a horse pathogen. The fact that SeMac does not inhibit opsonophagocytosis of $S$. equi by horse PMNs indicates that SeMac is not involved in the evasion of the acquired horse immunity against $S$. equi. This suggests that SeMac has other unknown function.

\section{ACKNOWLEDGEMENTS}

This work was supported by the National Research Initiative of the USDA Cooperative State Research, Education and Extension Service, grant number 2007-35204-18306), the National Center for Research Resources, grant P20 RR-020185, and the Montana State University Agricultural Experimental Station and USDA Formula Funds. We thank Drs. James Musser and Bettina Wagner for providing horse $S$. equi strains and purified horse IgG, respectively.

\section{REFERENCES}

[1] Timoney JF. Strangles. Vet Clin North Am Equine Pract 1993; 9: 365-74.

[2] Harrington DJ, Sutcliffe IC, Chanter N. The molecular basis of Streptococcus equi infection and disease. Microbes Infect 2002; 4: 501-10.

[3] Woolcock JB. Immunity to S. equi. Aust Vet J 1975; 51: 554-9.

[4] Timoney JF, Eggers D. Serum bactericidal responses to Streptococcus equi of horses following vaccination. Equine Vet J 1985; 17: 306-10

[5] Lei B, DeLeo FR, Hoe NP, et al. Evasion of human innate and acquired immunity by a bacterial homologue of CD11b that inhibits opsonophagocytosis. Nat Med 2001; 7: 1298-305.

[6] von Pawel-Rammingen U, Johansson BP, Björck L. IdeS, a novel streptococcal cysteine proteinase with unique specificity for immunoglobulin G. EMBO $J$ 2002; 21: 1607-15. 
[7] Lei B, DeLeo FR, Reid SD, et al. Opsonophagocytosis-inhibiting Mac protein of group A Streptococcus: identification and characteristics of two genetic complexes. Infect Immun 2002; 70: 6880-90.

[8] Lei B, Liu M, Meyers EG, Manning HM, Nagiec MJ, Musser JM histidine and aspartic acid residues important for $\operatorname{IgG}$ endopeptidase activity of the group A Streptococcus opsonophagocytosisinhibiting mac protein. Infect Immun 2003; 71: 2881-4.

[9] Agniswamy J, Lei B, Musser JM, Sun PD. Insight of host immune evasion mediated by two variants of Group A streptococcus Mac protein. J Biol Chem 2004; 279: 52789-96.

[10] Liu M, McClure MJ, Zhu H, Xie G, Lei B. The two-component regulatory system VicRK is important to virulence of Streptococcus equi subspecies equi. Open Microbiol J 2008; 2: 89-93.

[11] Hoe NP, Nakashima K, Grigsby D, et al. Rapid molecular genetic subtyping of serotype M1 group A Streptococcus strains. Emerg Infect Dis 1999; 5:254-263.

[12] Holden MT, Heather Z, Paillot R, et al. Genomic evidence for the evolution of Streptococcus equi: host restriction, increased virulence, and genetic exchange with human pathogens. PLoS Pathog 2009; 5: e1000346.

[13] White-Owen C, Alexander JW, Sramkoski RM, Babcock GF. Rapid whole-blood microassay using flow cytometry for measuring neutrophil phagocytosis. J Clin Microbiol 1992; 30: 2071-6.
[14] Liu M, Hanks TS, Zhang J, et al. Defects in ex vivo and in vivo growth and sensitivity to osmotic stress of group A Streptococcus caused by interruption of response regulator gene vicR. Microbiology 2006; 152: 967-78.

[15] Xie G, Liu M, Zhu H, Lei B. Esterase SeE of Streptococcus equi ssp. equi is a novel non-specific carboxylic ester hydrolase. FEMS Microbiol Lett 2008; 289: 181-6.

[16] Lei B, Mackie S, Lukomski S, Musser JM. Identification and immunogenicity of Group A Streptococcus culture supernatant proteins. Infect Immun 2000; 68: 6807-18.

[17] Wagner B. Immunoglobulins and immunoglobulin genes of the horse. Dev Comp Immunol 2006; 30:155-64.

[18] Lannergård J, Guss B. IdeE, an IgG-endopeptidase of Streptococcus equi ssp. equi. FEMS Microbiol Lett 2006; 262: 2305 .

[19] Timoney JF, Yang J, Liu J, Merant C. IdeE reduces the bactericidal activity of equine neutrophils for Streptococcus equi. Vet Immunol Immunopathol 2008; 122: 76-82.

[20] Heath A, DiRita VJ, Barg NL, Engleberg NC. A two-component regulatory system, CsrR-CsrS, represses expression of three Streptococcus pyogenes virulence factors, hyaluronic acid capsule, streptolysin S, and pyrogenic exotoxin B. Infect Immun 1999; 67: 5298-305.

(C) Liu and Lei; Licensee Bentham Open.

This is an open access article licensed under the terms of the Creative Commons Attribution Non-Commercial License (http://creativecommons.org/licenses/by$\mathrm{nc} / 3.0 /$ ) which permits unrestricted, non-commercial use, distribution and reproduction in any medium, provided the work is properly cited. 